\title{
Whitney arcs and 1-critical arcs
}

\author{
by \\ Marianna Csörnyei (London), Jan Kališ (Boca Raton, FL), \\ and Luděk Zajíček (Praha)
}

\begin{abstract}
A simple arc $\gamma \subset \mathbb{R}^{n}$ is called a Whitney arc if there exists a non-constant real function $f$ on $\gamma$ such that $\lim _{y \rightarrow x, y \in \gamma}|f(y)-f(x)| /|y-x|=0$ for every $x \in \gamma ; \gamma$ is 1-critical if there exists an $f \in C^{1}\left(\mathbb{R}^{n}\right)$ such that $f^{\prime}(x)=0$ for every $x \in \gamma$ and $f$ is not constant on $\gamma$. We show that the two notions are equivalent if $\gamma$ is a quasiarc, but for general simple arcs the Whitney property is weaker. Our example also gives an arc $\gamma$ in $\mathbb{R}^{2}$ each of whose subarcs is a monotone Whitney arc, but which is not a strictly monotone Whitney arc. This answers completely a problem of G. Petruska which was solved for $n \geq 3$ by the first author in 1999 .
\end{abstract}

1. Introduction. A famous example of Whitney [10] shows that there exist a simple arc $\gamma \subset \mathbb{R}^{2}$ and a $C^{1}$ function $f$ on $\mathbb{R}^{2}$ such that each point of $\gamma$ is critical for $f$, and $f$ is not constant on $\gamma$. A slightly weaker example was independently constructed by Choquet in [1]. Namely, he constructed a simple arc $\gamma \subset \mathbb{R}^{2}$ which is Whitney by the following terminology introduced in [8] and used in [4].

Definition 1.1. We say that a simple arc $\gamma \subset \mathbb{R}^{n}$ is a Whitney arc if there exists a non-constant real function $f$ on $\gamma$ such that

$$
\lim _{y \rightarrow x, y \in \gamma} \frac{|f(y)-f(x)|}{|y-x|}=0 \quad \text { for each } x \in \gamma \text {. }
$$

It seems that the difference between Whitney arcs thus defined and arcs considered by Whitney is not sufficiently emphasized in the literature (see e.g. remarks in [9, p. 399] on Choquet's results). The aim of the present article is to study this difference. First we recall the terminology of [9] which corresponds precisely to the example of Whitney.

2000 Mathematics Subject Classification: Primary 26B05; Secondary 26A30.

Key words and phrases: Whitney curve, quasiarc.

The third named author was supported by the grant MSM 0021620839 from the Czech Ministry of Education. 
Definition 1.2. We say that a simple arc $\gamma \subset \mathbb{R}^{n}$ is a 1 -critical arc if there exists a $C^{1}$ function on $\mathbb{R}^{n}$ which is not constant on $\gamma$ and $f^{\prime}(x)=0$ for each $x \in \gamma$.

Of course, each 1-critical arc is Whitney but the opposite implication does not hold. If the convergence in (1) were uniform in $x \in \gamma$ then Whitney's extension theorem would imply that $f$ can be extended to $\mathbb{R}^{n}$ as a $C^{1}$ function with derivative 0 at the points of $\gamma$; however, without assuming uniform convergence this is not the case. In Section 3 we will construct a Whitney arc $\gamma$ in $\mathbb{R}^{2}$ (slightly modifying the original construction of Whitney) which is not 1-critical.

No full characterization of 1-critical arcs or Whitney arcs is known (even in $\mathbb{R}^{2}$ ). However, there are interesting necessary or sufficient conditions. It is not difficult to prove (see [1] and Lemma 4.1 below) that no Whitney arc has $\sigma$-finite 1-dimensional Hausdorff measure. Choquet also proved that no graph of a continuous $f:[a, b] \rightarrow \mathbb{R}$ is Whitney. This result easily implies [5] that if $\gamma \subset \mathbb{R}^{n}$ has a parametrization whose $n-1$ coordinates have finite variation, then $\gamma$ is not a Whitney arc. Interesting necessary [8, Theorem 3] and sufficient [8, Theorem 2] conditions for $\gamma \subset \mathbb{R}^{n}$ to be Whitney were proved by Laczkovich and Petruska.

Norton [9] proved that each simple arc $\gamma$ in $\mathbb{R}^{n}$ which is a quasiarc and has Hausdorff dimension greater than 1 is 1-critical, and noted that such arcs "are in the plentiful supply (e.g. as Julia sets for certain rational maps in the plane)". (Note that all arcs constructed in [1], [4], [8] and [10] are quasiarcs.) We prove (Theorem 2.2) that if a Whitney arc in $\mathbb{R}^{n}$ is a quasiarc, then it is 1-critical. That is, for quasiarcs the two notions are equivalent.

A modification of the construction of Whitney (see Section 3) is used as a basic building block in an iterative construction in Section 4, which gives an example of a Whitney arc which is not 1-critical and also has other interesting properties. To describe them, recall that a real function $f$ defined (at least) on a simple arc $\gamma \subset \mathbb{R}^{n}$ is said to be monotone (resp. strictly monotone) along $\gamma$ if $f \circ \varphi$ is monotone (resp. strictly monotone) for each homeomorphic parametrization $\varphi$ of $\gamma$. Following [4], we say that a simple $\operatorname{arc} \gamma \subset \mathbb{R}^{n}$ is a monotone (resp. strictly monotone) Whitney arc if there exists a non-constant $f$ on $\gamma$ that is monotone (resp. strictly monotone) along $\gamma$ and satisfies (1).

Petruska raised the question whether there exists a simple arc $\gamma$ for which every subarc is Whitney, but for which there is no parametrization $\varphi$ of $\gamma$ satisfying

$$
\lim _{t \rightarrow t_{0}} \frac{\left|t-t_{0}\right|}{\left|\varphi(t)-\varphi\left(t_{0}\right)\right|}=0, \quad t_{0} \in[0,1]
$$

(which is clearly equivalent to $\gamma$ not being a strictly monotone Whitney arc). 
This question was answered affirmatively in [4] for $n \geq 3$, and it remained open in $\mathbb{R}^{2}$ (see Problem 4 in [4]). Our example gives an affirmative answer also for $n=2$. We construct an arc $\gamma \subset \mathbb{R}^{2}$ such that each of its subarcs is a monotone Whitney arc but any Lipschitz function satisfying (1) on any subarc $\gamma^{\prime}$ of $\gamma$ is constant on $\gamma^{\prime}$. From the last property it will easily follow that each function satisfying (1) on $\gamma$ is locally constant on a relatively open dense subset of $\gamma$ (and so $\gamma$ is not a strictly monotone Whitney arc).

For the sake of completeness we remark that Theorem 2.2 implies that every Whitney quasiarc is a monotone Whitney arc. However, if $\gamma$ is not a quasiarc then this is no longer true: Kolár $([6])$ recently constructed a 1-critical arc in $\mathbb{R}^{2}$ which is not a monotone Whitney arc (and since each 1-critical arc is a Whitney arc, this solves Problem 2 in [4]).

2. Whitney quasiarcs are 1-critical. We denote by $\lambda$ the Lebesgue measure on $\mathbb{R}$. In the following we will use the well-known notion of a quasiarc.

Definition 2.1. We say that a simple arc $\gamma \subset \mathbb{R}^{n}$ is a quasiarc if there exists $K>0$ such that, for any distinct $x, y \in \gamma$, the subarc of $\gamma$ "between $x$ and $y "$ (in the natural sense) is contained in some ball of radius $K|x-y|$.

Theorem 2.2. Let $\gamma \subset \mathbb{R}^{n}$ be a Whitney arc which is a quasiarc. Then there exists a $C^{1}$ function $f$ on $\mathbb{R}^{n}$ that is non-constant monotone along $\gamma$, and $f^{\prime}(x)=0$ for every $x \in \gamma$. In particular, $\gamma$ is 1-critical.

Proof. Let $\varphi:[0,1] \rightarrow \mathbb{R}^{n}$ be a continuous injective parametrization of $\gamma$. Choose a non-constant $f: \gamma \rightarrow \mathbb{R}$ such that (1) holds. We can suppose that $g:=f \circ \varphi$ is not non-increasing (otherwise we take $-f$ instead of $f$ ). So we can choose $0 \leq a<b \leq 1$ such that $g(a)<g(b)$. For each $y \in$ $[g(a), g(b)]$ put $\omega(y)=\min \{x \in[a, b]: g(x)=y\}$. Since $g$ is continuous, $\omega$ is clearly (strictly) increasing. Using Lusin's theorem and then the CantorBendixson theorem we can choose a set $T^{*} \subset[g(a), g(b)]$ such that $\lambda\left(T^{*}\right)>0$ and $\left.\omega\right|_{T^{*}}$ is continuous. Put $T:=\omega\left(T^{*}\right)$. Then $g_{0}:=\left.g\right|_{T}$ is an increasing homeomorphism between $T$ and $T^{*}$, and $g_{0}=\left.f_{0} \circ \varphi\right|_{T}$ where $f_{0}:=\left.f\right|_{\varphi(T)}$ is a homeomorphism between $\varphi(T)$ and $T^{*}$.

Let, for $x \in \gamma$,

$$
\eta_{k}(x):=\sup \left\{\frac{|f(y)-f(x)|}{|y-x|}: y \in \gamma, 0<|y-x|<1 / k\right\} .
$$

Then $\lim _{k \rightarrow \infty} \eta_{k}(x) \rightarrow 0$ for every $x \in \gamma$. It is easy to prove that $p_{k}:=$ $\eta_{k} \circ f_{0}^{-1}$ is a Borel function on $T^{*}$. Since $p_{k} \rightarrow 0$ at every point of $T^{*}$, applying Egorov's theorem (see $[3,2.3 .7]$ ) we can find a closed $H^{*} \subset T^{*}$ with $\lambda\left(H^{*}\right)>0$ such that $p_{k} \rightarrow 0$ uniformly on $H^{*}$. That is, the limit in (1) is uniform on $f_{0}^{-1}\left(H^{*}\right)$. 
Set $H:=g_{0}^{-1}\left(H^{*}\right)$. We can define a (strictly) increasing continuous function $\widetilde{g}$ on $[0,1]$ which extends $\left.g_{0}\right|_{H}$ and is linear on each component of $[0,1] \backslash H$. Put $q(t):=\lambda\left((-\infty, t] \cap H^{*}\right)$ and $F:=q \circ \widetilde{g} \circ \varphi^{-1}$. Then $F$ is a non-constant function monotone along $\gamma$. We will prove that

$$
\lim _{y \rightarrow x, x \in \gamma} \frac{|F(y)-F(x)|}{|y-x|}=0 \quad \text { uniformly with respect to } x \in \gamma .
$$

To this end consider an arbitrary $\varepsilon>0$. Let $K \geq 1$ witness the fact that $\gamma$ is a quasiarc. Note that $F=q \circ f$ on $\varphi(H)$ and $q$ is Lipschitz with constant 1, therefore $|F(y)-F(x)| \leq|f(y)-f(x)|$ for each $x, y \in \varphi(H)$. Using also the fact that the limit (1) is uniform with respect to $x \in \varphi(H)=f_{0}^{-1}\left(H^{*}\right)$, we can find $\delta>0$ such that

$$
\frac{|F(x)-F(y)|}{|x-y|}<\frac{\varepsilon}{2 K} \quad \text { whenever } x, y \in \varphi(H) \text { and } 0<|x-y|<\delta .
$$

Let $x, y \in \gamma$ be arbitrary points with $0<|x-y|<\delta(4 K)^{-1}$ and $F(x) \neq$ $F(y)$. We can suppose that $x=\varphi\left(t_{x}\right)$ and $y=\varphi\left(t_{y}\right)$ with $t_{x}<t_{y}$.

Since $F$ is constant on the intervals contiguous to $\varphi(H)$ and $F(x) \neq F(y)$, we see that $H$ has at least two points in $\left[t_{x}, t_{y}\right]$. Define

$$
s_{x}:=\min \left(H \cap\left[t_{x}, t_{y}\right]\right) \text { and } s_{y}=\max \left(H \cap\left[t_{x}, t_{y}\right]\right) .
$$

Clearly $t_{x} \leq s_{x}<s_{y} \leq t_{y}$ and $F$ is constant on $\varphi\left(\left[t_{x}, s_{x}\right]\right)$ and $\varphi\left(\left[s_{y}, t_{y}\right]\right)$. The definition of $K$ gives

$$
\left|\varphi\left(s_{x}\right)-\varphi\left(s_{y}\right)\right| \leq 2 K\left|\varphi\left(t_{x}\right)-\varphi\left(t_{y}\right)\right| \leq 2 K \frac{\delta}{4 K}<\delta
$$

and thus (3) gives

$$
\begin{aligned}
\frac{|F(x)-F(y)|}{|x-y|} & =\frac{\left|F\left(\varphi\left(t_{x}\right)\right)-F\left(\varphi\left(t_{y}\right)\right)\right|}{\left|\varphi\left(t_{x}\right)-\varphi\left(t_{y}\right)\right|} \leq \frac{\left|F\left(\varphi\left(s_{x}\right)\right)-F\left(\varphi\left(s_{y}\right)\right)\right|}{\frac{1}{2 K}\left|\varphi\left(s_{x}\right)-\varphi\left(s_{y}\right)\right|} \\
& <2 K \frac{\varepsilon}{2 K}=\varepsilon,
\end{aligned}
$$

which proves (2).

Whitney's extension theorem (see e.g. [2, p. 245]) and (2) immediately imply that there exists an extension $\widetilde{F}$ of $F$ such that $\widetilde{F} \in C^{1}\left(\mathbb{R}^{n}\right)$ and $(\widetilde{F})^{\prime}(x)=0$ for each $x \in \gamma$. Since $F$ is a non-constant monotone function along $\gamma$, we have proved Theorem 2.2 .

3. A modified Whitney's example: a Whitney arc which is not 1-critical. In this section we slightly modify the original construction of Whitney to obtain a class of Whitney arcs (called here MW-arcs for short) and prove some of their properties that are used in this section to give a simple construction of a Whitney arc which is not 1-critical, and are also used in Section 4 for constructing our main example. 
3.1. For the convenience of the reader we first repeat (almost word for word) the construction of Whitney from [10].

Let $Q=Q_{\emptyset}:=[0,1]^{2}$. Let $Q_{0}, Q_{1}, Q_{2}, Q_{3}$ be closed squares of side $1 / 3$ lying inside to $Q$ in clockwise order, each at distance 1/12 from the boundary of $Q$ as in Figure 1. Let $q$ and $q^{\prime}$ be the centres of the sides of $Q$ along $Q_{0}, Q_{1}$, and along $Q_{3}, Q_{0}$. Let $q_{i}$ and $q_{i}^{\prime}$ be the centres of two adjacent edges of $Q_{i}(i=0,1,2,3)$, as in Figure 1 . Let $A_{i}(i=0,1,2,3,4)$ be the line segments as in Figure 1.

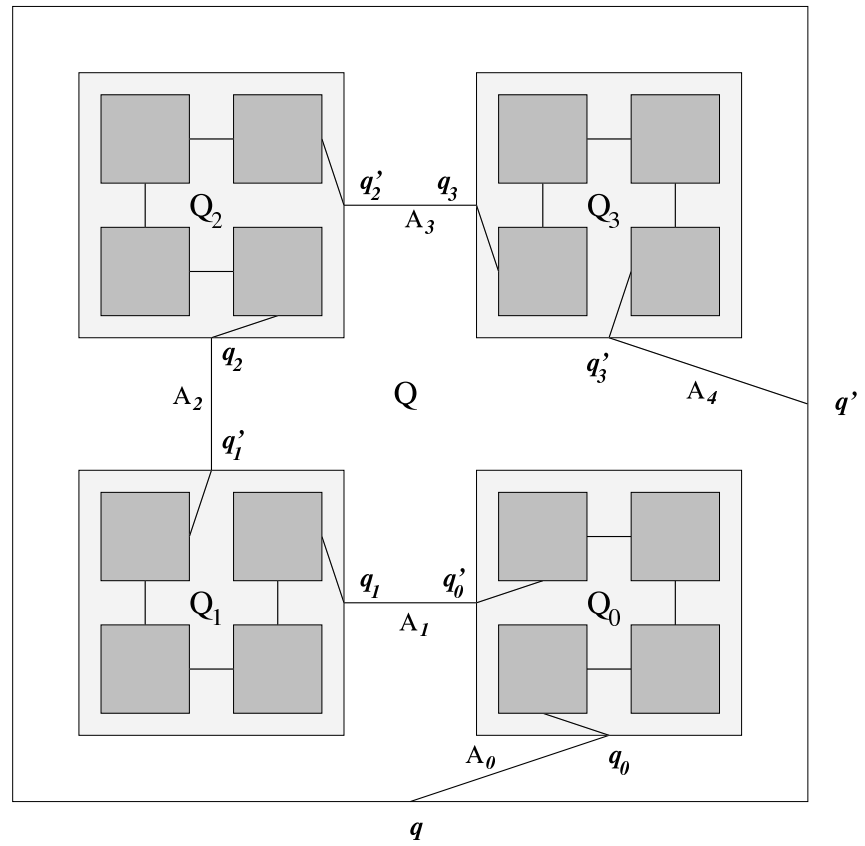

Fig. 1. Construction

Suppose we have constructed squares $Q_{i_{1} \ldots i_{t}}$, points $q_{i_{1} \ldots i_{t}}, q_{i_{1} \ldots i_{t}}^{\prime}$, and line segments $A_{i_{1} \ldots i_{t}, j}$ (each $\left.i_{k}=0,1,2,3 ; j=0,1,2,3,4\right)$ for $t<s$. By taking a square $Q_{i_{1} \ldots i_{s-2}}$, shrinking it to a third of its size, and turning it around and upside down if necessary, we may place it in $Q_{i_{1} \ldots i_{s-1}}$ so that $q_{i_{1} \ldots i_{s-2}}$ and $q_{i_{1} \ldots i_{s-2}}^{\prime}$ go into $q_{i_{1} \ldots i_{s-1}}$ and $q_{i_{1} \ldots i_{s-1}}^{\prime}$, and thus construct four new squares $Q_{i_{1} \ldots i_{s}}\left(i_{s}=0,1,2,3\right)$ as images of $Q_{i_{1} \ldots i_{s-2} i_{s}}$, furthermore points $q_{i_{1} \ldots i_{s}}$, $q_{i_{1} \ldots i_{s}}^{\prime}$ and segments $A_{i_{1} \ldots i_{s-1} j}$ for $j=0,1,2,3,4$ as images of $q_{i_{1} \ldots i_{s-2} i_{s}}$, $q_{i_{1} \ldots i_{s-2} i_{s}}^{\prime}$ and $A_{i_{1} \ldots i_{s-2} j}$, respectively. We denote the point $Q \cap Q_{i_{1}} \cap Q_{i_{1} i_{2}} \cap \ldots$ by $Q_{i_{1} i_{2} \ldots}$.

It is not difficult to see that the line segments $A_{i_{1} \ldots i_{s}}$ together with the points $Q_{i_{1} i_{2} \ldots}$ form a simple arc $A$ (a canonical parametrization is described in $[10])$. 
Now define $F$ on $A$ as follows:

$$
F(x):= \begin{cases}\frac{i_{1}}{4}+\frac{i_{2}}{4^{2}}+\cdots+\frac{i_{s}}{4^{s}}, & x \in A_{i_{1} i_{2} \ldots i_{s}}, \\ \frac{i_{1}}{4}+\frac{i_{2}}{4^{2}}+\cdots, & x=Q_{i_{1} i_{2} \ldots} .\end{cases}
$$

Whitney proved that $F$ is a restriction of a $C^{1}$ function $F^{*}$ defined on the plane such that each point of $A$ is critical for $F^{*}$.

3.2. Now we will make some modifications which lead to a class of Whitney (but not 1-critical) arcs.

In the following, the symbol $\underline{i}$ will always denote a sequence $\underline{i}=i_{1} \ldots i_{k}$ where $i_{n} \in\{0,1,2,3\}$ (for $k=0$ we set $\underline{i}=\emptyset$ ); we define $|\underline{i}|:=k$. For each $\underline{i}=i_{1} \ldots i_{k}$ and $j \in\{0,1,2,3,4\}$ we choose an arbitrary simple arc $\gamma_{\underline{i}, j}$ lying (except the endpoints) in int $Q_{\underline{i}} \backslash\left(Q_{\underline{i} 0} \cup Q_{\underline{i} 1} \cup Q_{\underline{i} 2} \cup Q_{\underline{i} 3}\right)$ that connects the same points as $A_{\underline{i}, j}$, such that the arcs $\gamma_{\underline{i}, j}$ are pairwise disjoint and

$$
\operatorname{dist}\left(\gamma_{\underline{i}, j}, \gamma_{\underline{i}, j+1}\right)<1 / 5^{k}, \quad j=0,1,2,3 .
$$

It is easy to show that the $\operatorname{arcs} \gamma_{\underline{i}, j}$ together with the points $Q_{i_{1} i_{2} \ldots}$ form a simple arc $\gamma$. We will choose points $a_{\underline{i}, j}, b_{\underline{i}, j} \in \gamma_{\underline{i}, j}$ such that

$$
\operatorname{dist}\left(a_{\underline{i}, j}, b_{\underline{i}, j+1}\right)<1 / 5^{k}, \quad j=0,1,2,3 .
$$

We will call any arc constructed in this way an $M W$-arc (that is, an arc obtained by the modified Whitney construction).

3.3. We show that each MW-arc $\gamma$ is a monotone Whitney arc. To this end consider the function $f$ on $\gamma$ which agrees with $F$ at the points $Q_{i_{1} i_{2} \ldots}$ and is constant on each $\gamma_{\underline{i}, j}$ with the same value as $F$ has on $A_{\underline{i}, j}$. Clearly $f$ is monotone along $\gamma$. We will show that (1) holds. It is immediate that (1) holds at the points of the $\operatorname{arcs} \gamma_{\underline{i}, j}$, since $f$ is constant on these arcs and each such arc has, in the space $\gamma$, a neighbourhood formed by three (or two) $\operatorname{arcs} \gamma_{\underline{i}, j}$. Now let $x=Q_{i_{1} i_{2} \ldots}$ and let $y$ be an arbitrary point of $\gamma$ different from $x$. Consider the largest $k$ with $y \in Q_{i_{1} \ldots i_{k}}=Q_{\underline{i}}$. Then we can see that $|f(x)-f(y)| \leq 1 / 4^{k}$, while $|x-y| \geq \operatorname{dist}\left(Q_{\underline{i} i_{k+1} i_{k+2}}, \partial Q_{\underline{i} i_{k+1}}\right)=1 /\left(12 \cdot 3^{k+1}\right)$. This shows

$$
\lim _{y \rightarrow x, y \in \gamma} \frac{|f(y)-f(x)|}{|y-x|} \leq \lim _{k \rightarrow \infty} \frac{12 \cdot 3^{k+1}}{4^{k}}=0 .
$$

3.4. Now we will show that if $f$ is a Lipschitz function on an MW-arc $\gamma$, then

$$
\lambda(f(\gamma)) \leq \sum_{\underline{i}, 0 \leq j \leq 4} \lambda\left(f\left(\gamma_{\underline{i}, j}\right)\right)
$$


Let $f$ be Lipschitz with constant $K$. For each $k \in \mathbb{N}$, let

$$
I_{k}:=\bigcup_{|\underline{i}| \leq k, 0 \leq j \leq 4} f\left(\gamma_{\underline{i}, j}\right) \cup \bigcup_{|\underline{i}|=k, 0 \leq j \leq 3}\left[f\left(a_{\underline{i}, j}\right), f\left(b_{\underline{i}, j+1}\right)\right] .
$$

It is easy to see that $I_{k}$ is a closed interval, since it is clearly connected and closed; let $I_{k}=:[u, v]$. Now observe that $f(\gamma) \subset\left[u-K \sqrt{2} / 3^{k+1}, v+\right.$ $\left.K \sqrt{2} / 3^{k+1}\right]$. This follows by the Lipschitz property of $f$, the definition of $I_{k}$ and the obvious fact that $\operatorname{dist}\left(c, \bigcup_{|\underline{i}| \leq k, 0 \leq j \leq 4} \gamma_{\underline{i}, j}\right) \leq \sqrt{2} / 3^{k+1}$ for every $c \in \gamma$.

Clearly

$$
\lambda\left(I_{k} \backslash \bigcup_{|\underline{i}| \leq k, 0 \leq j \leq 4} f\left(\gamma_{\underline{i}, j}\right)\right) \leq \sum_{|\underline{i}|=k, 0 \leq j \leq 3}\left|f\left(b_{\underline{i}, j+1}\right)-f\left(a_{\underline{i}, j}\right)\right| \leq \frac{K 4^{k+1}}{5^{k}} .
$$

Therefore

$$
\lambda(f(\gamma)) \leq \sum_{\underline{i}, 0 \leq j \leq 4} \lambda\left(f\left(\gamma_{\underline{i}, j}\right)\right)+\frac{K 4^{k+1}}{5^{k}}+2 K \sqrt{2} / 3^{k+1},
$$

which easily implies (6).

Similarly to (6), we find that for each $\underline{i}^{*}=i_{1}^{*} \ldots i_{s}^{*}$,

$$
\lambda\left(f\left(\gamma \cap Q_{\underline{i}^{*}}\right)\right) \leq \sum_{\underline{i}, 0 \leq j \leq 4} \lambda\left(f\left(\gamma_{\underline{i}, j} \cap Q_{\underline{i}^{*}}\right)\right) .
$$

3.5. Now we can prove the following result:

THEOREM 3.1. There exists a Whitney arc $\gamma \subset \mathbb{R}^{2}$ which is not 1critical. Moreover, there exists no non-constant Lipschitz function $f$ on $\gamma$ which satisfies (1).

Proof. We choose $\gamma$ as an arbitrary MW-arc for which all the $\operatorname{arcs} \gamma_{\underline{i}, j}$ are polygons. Thus $\gamma$ is a (monotone) Whitney arc.

Now suppose that $f$ is a Lipschitz function on $\gamma$ which satisfies (1) on $\gamma$. Then, since a polygon is not a Whitney arc, $\lambda\left(f\left(\gamma_{\underline{i}, j}\right)\right)=0$ for each arc $\gamma_{\underline{i}, j}$ and hence (6) implies that $\lambda(f(\gamma))=0$ and thus $f$ is constant on $\gamma$. Since each $C^{1}$ function on $\mathbb{R}^{2}$ is Lipschitz on $\gamma$, we have proved that the arc $\gamma$ is not 1-critical.

4. The main example. We will need the following result (see [1, p. 49]).

Lemma 4.1. Suppose that $A \subset \mathbb{R}^{n}$ has $\sigma$-finite one-dimensional Hausdorff measure and $f$ is a real function on $A$ such that

$$
\lim _{y \rightarrow x, y \in A} \frac{|f(y)-f(x)|}{|y-x|}=0 \quad \text { for each } x \in A .
$$

Then $\lambda(f(A))=0$. 
Using the generalized Whitney construction from Section 3 we will now prove the following main result of the present article.

TheOREM 4.2. There exists a simple arc $\gamma \subset \mathbb{R}^{2}$ such that:

(i) Each subarc of $\gamma$ is a monotone Whitney arc.

(ii) There is no non-constant Lipschitz function $f$ on any subarc $\gamma^{*}$ of $\gamma$ such that $f$ satisfies (1) on $\gamma^{*}$.

(iii) Each function satisfying (1) on $\gamma$ is locally constant on a relatively open dense subset of $\gamma$. In particular, $\gamma$ is not a strictly monotone arc.

Proof. First note that (iii) is an easy consequence of (ii). Indeed, suppose that (ii) holds, $f$ satisfies (1) on $\gamma$, and $\gamma^{*}$ is an arbitrary subarc of $\gamma$. For each $n \in \mathbb{N}$, let $Z_{n}$ denote the set of all $x \in \gamma^{*}$ such that $|f(y)-f(x)| \leq|y-x|$ whenever $y \in \gamma$ and $|y-x| \leq 1 / n$. Since each $Z_{n}$ is closed and $\gamma^{*}=\bigcup Z_{n}$, the Baire category theorem implies that there exists $n \in \mathbb{N}$ and a subarc $\gamma^{* *}$ of $\gamma^{*}$ with diam $\gamma^{* *}<1 / n$ and $\gamma^{* *} \subset Z_{n}$. Then $f$ is Lipschitz on $\gamma^{* *}$ and thus constant on $\gamma^{* *}$ by (ii), and (iii) follows.

Now we fix an arbitrary MW-arc $\widetilde{\gamma}$ for which all the arcs $\widetilde{\gamma}_{i, j}$ are polygons and we will construct $\gamma$ by an iterative procedure, as follows.

SteP 1. Let $\gamma^{1}:=\widetilde{\gamma}$. We choose a countable set $\mathcal{Q}^{1}$ of disjoint closed squares such that each square in $\mathcal{Q}^{1}$ is inside $Q_{\underline{i}} \backslash\left(Q_{\underline{i} 0} \cup Q_{\underline{i} 1} \cup Q_{\underline{i 2}} \cup Q_{\underline{i} 3}\right)$ for some $\underline{i}$, it meets precisely one arc $\widetilde{\gamma}_{\underline{i}, j}$, and its intersection with $\widetilde{\gamma}_{\underline{i}, j}$ is a line segment that connects the centres of two adjacent edges of the square. We also require that $\bigcup \mathcal{Q}^{1}$ covers a dense subset of $\bigcup_{\underline{i}, j} \widetilde{\gamma}_{\underline{i}, j}$,

$$
\begin{gathered}
\text { no point } a_{\underline{i}, j} \text { or } b_{\underline{i}, j} \text { (cf. (5)) is contained in } \bigcup \mathcal{Q}^{1} \text { and } \\
r:=\sum_{Q^{*} \in \mathcal{Q}^{1}} \text { edge length of } Q^{*}<1 .
\end{gathered}
$$

Step 1 concludes with the arc $\gamma^{1}=\widetilde{\gamma}$ and the set of squares $\mathcal{Q}^{1}$. For any $m \geq 1$, the $m$ th step will conclude with a simple arc $\gamma^{m}$ and a set of disjoint squares $\mathcal{Q}^{m}$ such that $\gamma^{m}$ intersects each square $Q^{*} \in \mathcal{Q}^{m}$ in a line segment that connects the centres of two adjacent edges of $Q^{*}$. Observe that, using (8), we easily deduce that

$$
\text { any simple arc } \eta \subset \cup \mathcal{Q}^{1} \cup \gamma^{1} \text { such that } \eta \backslash \bigcup \mathcal{Q}^{1}=\gamma^{1} \backslash \bigcup \mathcal{Q}^{1} \text { is an }
$$
MW-arc.

SteP $m$. Suppose that $\gamma^{m-1}$ and $\mathcal{Q}^{m-1}$ have been defined. We will repeat the same construction as in Step 1 inside each of the squares of $\mathcal{Q}^{m-1}$ :

For each $Q^{*} \in \mathcal{Q}^{m-1}$ choose a similarity $\psi_{Q^{*}}$ of the plane that maps the unit square $Q=[0,1]^{2}$ onto $Q^{*}$, such that the segment between $q$ and $q^{\prime}$ is 
mapped onto the segment $Q^{*} \cap \gamma^{m-1}$. Let

$$
\begin{aligned}
\gamma^{m} & =\left(\gamma^{m-1} \backslash \bigcup \mathcal{Q}^{m-1}\right) \cup \underset{Q^{*} \in \mathcal{Q}^{m-1}}{\bigcup} \psi_{Q^{*}}\left(\gamma^{1}\right), \\
\mathcal{Q}^{m} & =\left\{\psi_{Q^{*}}(\widetilde{Q}): Q^{*} \in \mathcal{Q}^{m-1}, \widetilde{Q} \in \mathcal{Q}^{1}\right\} .
\end{aligned}
$$

It is easy to see by induction on $m$ that

$$
r^{m}=\sum_{Q^{*} \in \mathcal{Q}^{m}} \text { edge length of } Q^{*} \text {. }
$$

Let $\gamma:=\bigcap_{m=1}^{\infty}\left(\gamma^{m} \cup \bigcup \mathcal{Q}^{m}\right)$. It is geometrically obvious and not difficult to prove that $\gamma$ is a simple arc. For a precise proof we have at least two possibilities. The more straightforward one is to define inductively "natural" parametrizations of $\gamma^{m}$ and to check that the limit of these parametrizations is an injective parametrization of $\gamma$. The other possibility is to apply [7, Theorem 3, Section V, §47] which gives a sufficient condition for a set to be a simple arc, which is rather easy to verify for our set $\gamma$. (We choose $C_{n}:=\gamma^{n} \cup \bigcup \mathcal{Q}^{n}$; for the definition of $A_{n}$ and $B_{n}$ we use the natural order on $\gamma^{n}$.)

Using (10), we find that $\gamma$ is an MW-arc. Also, for each $Q^{*} \in \bigcup_{m=1}^{\infty} \mathcal{Q}^{m}$, we infer by (10) that

$$
\psi_{Q^{*}}^{-1}\left(\gamma \cap Q^{*}\right) \text { is an MW-arc }
$$

and therefore $\gamma \cap Q^{*}$ is a monotone Whitney arc. Therefore each subarc of $\gamma$ is a monotone Whitney arc.

For each $Q^{*} \in \bigcup_{m=1}^{\infty} \mathcal{Q}^{m}$, let $\widetilde{\gamma}_{\underline{i}, j, Q^{*}}:=\psi_{Q^{*}}\left(\widetilde{\gamma}_{\underline{i}, j}\right)$ and $\gamma_{\underline{i}, j, Q^{*}}$ be the subarc of $\gamma$ with the same endpoints as $\widetilde{\gamma}_{\underline{i}, j, Q^{*}}$.

To prove (ii), first suppose that $f: \gamma \rightarrow \mathbb{R}$ is a Lipschitz function defined on the whole arc $\gamma$ that satisfies (1). Let $K$ denote the Lipschitz constant of $f$.

Consider an arbitrary $Q \in \mathcal{Q}^{k}$ and an arbitrary $\operatorname{arc} \gamma_{\underline{i}, j, Q}$. Since

$$
\gamma_{\underline{i}, j, Q}=\left(\gamma_{\underline{i}, j, Q} \cap \widetilde{\gamma}_{\underline{i}, j, Q}\right) \cup \bigcup_{Q^{*} \in \mathcal{Q}^{k+1}, Q^{*} \cap \gamma_{\underline{i}, j, Q} \neq \emptyset}\left(Q^{*} \cap \gamma\right)
$$

and $\gamma_{\underline{i}, j, Q} \cap \widetilde{\gamma}_{\underline{i}, j, Q}$ is rectifiable, Lemma 4.1 implies $\lambda\left(f\left(\gamma_{\underline{i}, j, Q} \cap \widetilde{\gamma}_{\underline{i}, j, Q}\right)\right)=0$ and therefore

$$
\lambda\left(f\left(\gamma_{\underline{i}, j, Q}\right)\right) \leq \sum_{Q^{*} \in \mathcal{Q}^{k+1}, Q^{*} \cap \gamma_{\underline{\underline{w}}, j, Q} \neq \emptyset} \lambda\left(f\left(Q^{*} \cap \gamma\right)\right) .
$$

By (12) and (6) we obtain

$$
\lambda(f(Q \cap \gamma)) \leq \sum_{\underline{i}, j} \lambda\left(f\left(\gamma_{\underline{i}, j, Q}\right)\right) .
$$


Using also (13) we obtain

$$
\lambda(f(Q \cap \gamma)) \leq \sum_{Q^{*} \subset Q, Q^{*} \in \mathcal{Q}^{k+1}} \lambda\left(f\left(Q^{*} \cap \gamma\right)\right) .
$$

Using this inequality and (11), we conclude by induction that, for any $m \in \mathbb{N}$,

$$
\lambda(f(\gamma)) \leq \sum_{Q^{*} \in \mathcal{Q}^{m}} \lambda\left(f\left(Q^{*} \cap \gamma\right)\right) \leq K \sum_{Q^{*} \in \mathcal{Q}^{m}} \operatorname{diam} Q^{*} \leq K \sqrt{2} r^{m}
$$

and therefore $\lambda(f(\gamma))=0$.

Now let $f: \gamma^{*} \rightarrow \mathbb{R}$ be a Lipschitz function defined on a subarc $\gamma^{*}$ of $\gamma$ that satisfies (1) on $\gamma^{*}$. If $\gamma^{*}$ is of the form $\gamma^{*}=\gamma \cap T$, where $T$ is a square of the form $T=\psi_{Q^{*}}\left(Q_{\underline{i}}\right)$ (where $\underline{i}$ is a finite sequence (possibly empty), $Q^{*} \in \bigcup_{m=0}^{\infty} \mathcal{Q}^{m}, \mathcal{Q}^{0}:=\left\{Q_{\emptyset}=[0,1]^{2}\right\}$ and $\psi_{Q_{\emptyset}}$ is the identity), then we deduce that $f$ is constant using (7) and the same argument as above for $\gamma^{*}=\gamma$. A general $\gamma^{*}$ can be written as a union of countably many subarcs of the above form and a $\sigma$-rectifiable set. Indeed, consider any point $x \in \gamma^{*}$ which is not an endpoint of $\gamma^{*}$. If $x \in \bigcup \mathcal{Q}^{m}$ for every $m$ then $\{x\}=\bigcap_{m=1}^{\infty} Q^{m}$ for some $Q^{m} \in \mathcal{Q}^{m}$, and if $m$ is large enough then $x \in Q^{m} \cap \gamma=\psi_{Q^{m}}\left(Q_{\emptyset}\right) \cap \gamma \subset \gamma^{*}$. If $x$ is not of this form, then there is a largest $m$ so that $x \in Q^{*}$ for some $Q^{*} \in \bigcup \mathcal{Q}^{m}$. Then $x \in \psi_{Q^{*}}(\widetilde{\gamma})$, therefore either $x=\psi_{Q^{*}}\left(Q_{i_{1} i_{2} \ldots}\right)$ for an infinite sequence $i_{1} i_{2} \ldots$ (in which case $x \in \psi_{Q^{*}}\left(Q_{i_{1} i_{2} \ldots i_{s}}\right) \cap \gamma \subset \gamma^{*}$ if $s$ is sufficiently large), or $x \in \psi_{Q^{*}}\left(\widetilde{\gamma}_{\underline{i}, j}\right)$ for some $\underline{i}, j$ (and $\psi_{Q^{*}}\left(\widetilde{\gamma}_{\underline{i}, j}\right)$ is a polygon, therefore it is rectifiable).

Thus, using also Lemma 4.1, we obtain $\lambda\left(f\left(\gamma^{*}\right)\right)=0$ and so $f$ is constant on $\gamma^{*}$.

5. Notes on $k$-critical arcs. The following definition is used in [9].

Definition 5.1. We say that a simple arc $\gamma \subset \mathbb{R}^{n}$ is $k$-critical if there exists a $C^{k}$ function on $\mathbb{R}^{n}$ which is not constant on $\gamma$ and $f^{\prime}(x)=0$ for each $x \in \gamma$.

The following related notion was implicitly used in [10].

Definition 5.2. We say that a simple arc $\gamma \subset \mathbb{R}^{n}$ is $k^{*}$-critical if there exists a $C^{k}$ function on $\mathbb{R}^{n}$ which is not constant on $\gamma$ and $f^{(j)}(x)=0$ for each $x \in \gamma$ and $1 \leq j \leq k$.

Note that the Morse-Sard theorem implies that there is no $k$-critical arc in $\mathbb{R}^{n}$ for $k \geq n$. On the other hand, Whitney [10, p. 517] has showed how the (above) planar construction can be generalized to obtain an $(n-1)^{*}$-critical $\operatorname{arc}$ in $\mathbb{R}^{n}$.

The following notion is implicitly used in [1]. 
Definition 5.3. We say that a simple arc $\gamma \subset \mathbb{R}^{n}$ is $k_{*}$-critical if there exists a non-constant real function $f$ on $\gamma$ such that

$$
\lim _{y \rightarrow x, y \in \gamma} \frac{|f(y)-f(x)|}{|y-x|^{k}}=0 \quad \text { for each } x \in \gamma .
$$

Note that Choquet [1] observed that no $k_{*}$-critical arc has $\sigma$-finite $k$ dimensional Hausdorff measure (cf. [5], where also some sufficient and some necessary conditions are presented).

Proceeding as in the proof of Theorem 2.2, we easily obtain a generalization.

TheOREM 5.4. Let $\gamma \subset \mathbb{R}^{n}$ be a $k_{*}$-critical arc which is a quasiarc. Then there exists a $C^{k}$ function $f$ on $\mathbb{R}^{n}$ such that $f$ is a non-constant monotone function along $\gamma$, and $f^{\prime}(x)=\cdots=f^{(k)}(x)=0$ for every $x \in \gamma$. In particular, $\gamma$ is $k^{*}$-critical and thus also $k$-critical.

Modifying the above mentioned Whitney construction of an $(n-1)^{*}$ critical arc in $\mathbb{R}^{n}$ in the same way as in the proof of Theorem 3.1, we obtain the following result.

TheOREM 5.5. There exists an $(n-1)_{*}$-critical arc $\gamma$ in $\mathbb{R}^{n}$ which is not 1-critical. Moreover, there exists no non-constant Lipschitz function $f$ on $\gamma$ which satisfies (1).

We do not know whether each $k$-critical arc is $k^{*}$-critical.

\section{References}

[1] G. Choquet, L'isométrie des ensembles dans ses rapports avec la théorie du contact et la théorie de la mesure, Mathematica (Timişoara) 20 (1944), 29-64.

[2] L. Evans and R. Gariepy, Measure Theory and Fine Properties of Functions, Stud. Adv. Math., CRC Press, Boca Raton, 1992.

[3] H. Federer, Geometric Measure Theory, Grundlehren Math. Wiss. 153, Springer, New York, 1969.

[4] M. Csörnyei, On Whitney pairs, Fund. Math. 160 (1999), 63-79.

[5] J. Kališ, On Whitney sets and their generalization, Real Anal. Exchange 30 (20042005), 385-392.

[6] J. Koláŕ, private communication.

[7] K. Kuratowski, Topology II, Academic Press, New York, 1968.

[8] M. Laczkovich and G. Petruska, Whitney sets and sets of constancy. On a problem of Whitney, Real Anal. Exchange 10 (1984-85), 313-323.

[9] A. Norton, Functions not constant on fractal quasi-arcs of critical points, Proc. Amer. Math. Soc. 106 (1989), 397-405.

[10] H. Whitney, A function not constant on a connected set of critical points, Duke Math. J. 1 (1935), 514-517. 
Department of Mathematics

University College London

Gower Street, London

WC1E 6BT, United Kingdom

E-mail:mari@math.ucl.ac.uk

Department of Mathematical Analysis

Charles University

Sokolovská 83

18675 Praha 8, Czech Republic

E-mail: zajicek@karlin.mff.cuni.cz
Department of Mathematical Sciences

Florida Atlantic University

777 Glades Road

Boca Raton, FL 33431, U.S.A.

E-mail: kalis@math.fau.edu

Received 30 September 2005;

in revised form 11 January 2007 